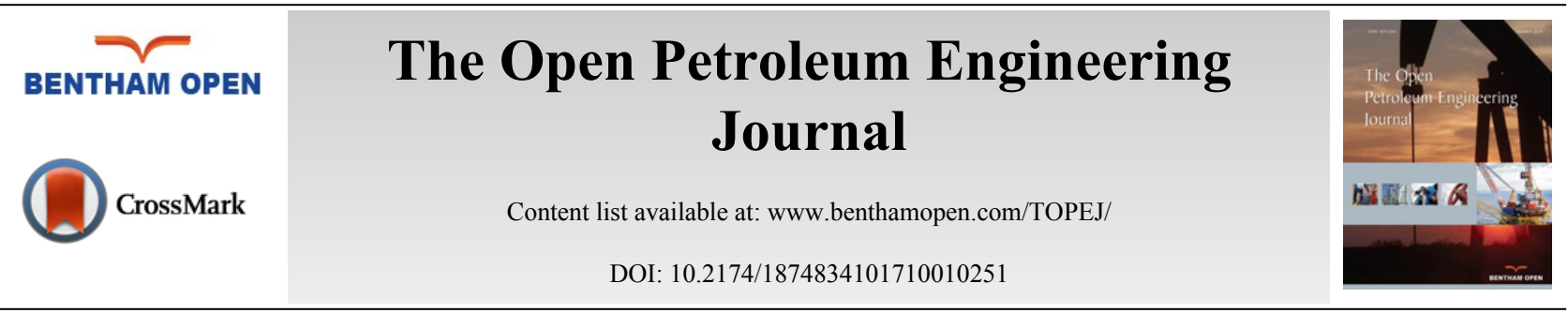

\title{
LETTER
}

\section{RDR Application: An Accurate HTHP Rheological Modeling for The Sulphonated Water-Based Drilling Fluid}

\author{
Lin $\mathrm{Xu}^{1,3, *}, \mathrm{Han} \mathrm{Gao}^{2}$, Ming-biao $\mathrm{Xu}^{3}, \mathrm{Fu}-$ chang $\mathrm{You}^{4}$ and Xiao-liang $\mathrm{Wang}^{4}$ \\ ${ }^{I}$ College of Petrochemical and Energetic Engineering, Zhejiang Ocean University, Zhoushan 316022, P.R China \\ ${ }^{2}$ Engineering Technology Research Institute of PetroChina Xinjiang Oilfield Company, Karamay 834000, P.R China \\ ${ }^{3}$ Hubei Collaborative Innovation Center for Unconventional Oil and Gas, Wuhan 430100, P.R China \\ ${ }^{4}$ Jiahua Technology Co., Ltd, Jingzhou 434000, P.R China
}

Received: March 24, 2017

Revised: June 28, 2017

Accepted: October 03,2017

\section{Abstract:}

\section{Introduction:}

An accurate HTHP rheological model of drilling fluids is critical for the safe and economic drilling operation. However, general HTHP rheological modeling methods appear to be very limited in the predictive accuracy.

\section{Materials and Method:}

In this work, a particular relative dial readings(RDR) modeling experiment was conducted on a weighted sulphonated water-based drilling fluid within a certain temperature and pressure range $\left(30-150^{\circ} \mathrm{C}, 0.1-100 \mathrm{MPa}\right)$, in combination to dial reading data of six specific shear rates 3,6,100,200,300, and 600rpm, to develop a highly accurate HTHP rheological model. The RDR modeling procedure was investigated in details, including relative dial reading, Arrhenius relation, polynomial of pressure coefficients, and polynomial of shear rate coefficients. An equation relating RDR to temperature, pressure, and shear rate was determined.

\section{Results:}

The predictive deviation was calculated to be lower than $11.7 \%$, and large errors occurred under the conditions of high pressure and low shear rates; all of which meet the requirement of in-field predictive accuracy. These results not only provide an accurate HTHP rheological equation for the weighted sulphonated water-based drilling fluid, but also propose an effective HTHP rheological modeling strategy for drilling fluids.

Keywords: RDR, HTHP, Rheological model, The sulphonated water-based drilling fluids, Predictive deviation.

\section{INTRODUCTION}

The rheological properties of drilling fluids are always critical upon drilling. Reasonable control of rheology can direct some key operation designs such as hydraulic calculation, pressure loss calculation, hole cleaning efficiency, and equivalent circulating density(ECD) determination, which enable high rate of penetration(ROP) and low drilling cost. The determination of rheological data relies, to a large extent, on a rheological mathematic representation, which allows an accurate theoretical prediction. Therefore, the rheological modeling of drilling fluids usually behaves as the first step especially in the high temperature high pressure(HTHP) drilling operation.

However, it is well-known that in deep drilling operations, rheology of drilling fluid is relatively complicated and often influenced by temperature, pressure, shear history, and composition of the drilling fluids [1 - 5]. Otherwise,

\footnotetext{
* Address correspondence to this author at the College of Petrochemical and Energetic Engineering, Zhejiang Ocean University, No. 1, South Haida Road, Zhoushan, 316022, P.R. China; Tel: +8613567661648; Fax: +867168060863, E-mail: xuhu_11@yeah.net
} 
effects of temperature and pressure on rheology of drilling fluids appear to be reverse in the down-hole conditions. Not surprisingly, developing a correlation that will relate shear stress to temperature, pressure, and shear rate should be difficult for drilling fluids. In the last 20-30 years, two important HTHP rheological modeling strategies, i.e., multiplicative factor(MF) and relative dial reading(RDR) approach, have been developed. But only limited studies on these two modeling application have been referred. As for the MF modeling approach, it is necessary to firstly fit the actual dial reading data to a general rheological model; while for the RDR approach, the relation that relates dial reading(shear stress) of a particular fluid at different temperature, pressure, and shear rate conditions can be directly determined. Undoubtedly, the latter approach should be more accurate because of avoidance of general model assumption, when compared to the former one.

In this work, the weighted sulphonated water-based fluid was used to explore applicability of the RDR modeling approach in combination to operation environments in Southwest China Gas Fields. To shed more light on HTHP rheological modeling, development of HTHP rheological model of drilling fluids will be further illustrated below.

\subsection{General HTHP Rheological Modeling Process}

The general rheological modeling process involves two steps: one is to fit dial reading(or shear stress) and shear rate values to specific rheological equations(e.g., Yield Power Law and Bingham Plastic models); the other is to determine the effect of temperature and pressure on dial readings and define correction factors to correlate rheological parameters in down-hole conditions. The commonly reported correction factor is shown as follows:

$$
f(P, T)=\exp \left(\frac{A+(B T+C) P}{T}\right)
$$

where A, B, and C are characteristic constants. Following Eq.(1), McMordie et al. [6] modified the power-law model as,

$$
\ln \tau=\ln K^{\prime}+n \ln \gamma+A P+B / T
$$

where $K$ ' and $n$ correspond to the consistency index and behavior index, respectively. This HTHP rheological model has been successfully used in the oil-based drilling fluid. Similarly, Houwen [7], Hiller [8], and Alderman [9] have applied the MF method to the two-parameter Casson and Bingham plastic models, as well as three-parameter HurschelBulkley and Robertson-Stiff models. On the basis of modeling procedure and results, they concluded that the correlation of parameters in base equations can disturb the final predictive accuracy.

To diminish the correlation of parameters, Rommetveit et al. [10] proposed another way to construct base equation, which is written as,

$$
f(P, T, \gamma)=e^{g_{1}(P, T)} \gamma+e^{g_{2}(P, T)}
$$

where $f(P, T, \gamma)$ is a factor that multiplies shear stress obtained at considered conditions. The constant functions $g_{I}(P, T)$ and $g_{2}(P, T)$ are determined by an optimistic fit to measured data. In this case, the intrinsic defects of the selected base models can be avoided, which makes prediction more accurate.

American Petroleum Institute(API) recommends the HTHP rheological model:

$$
\tau(T, P, \gamma)=\tau_{o}(\gamma) e^{\left[\alpha\left(T-T_{o}\right)+\beta\left(P-P_{o}\right)\right]}
$$

where $\tau_{o}$ denotes the shear stress at a given rotational speed. This model is extended to viscosity expressions [11, $12]$.

It should be noted that upon modeling, general HTHP rheological models depend on the initial base equation and Arrhenius empirical relations, which can cause a large predictive deviation.

\subsection{Specific HTHP Rheological Modeling Process}

To avoid designation of initial rheological model, a specific HTHP rheological modeling process based on the RDR approach was developed by Hemphill [13]. Rather than using a designated rheological model, it directly correlated the changes in dial readings with temperature, pressure, and shear rate by the non-linear regression method. All the results 
were related to a relative parameter measured at $T_{o}$ and $P_{o}$. The RDR are defined as:

$$
R D R=\frac{\theta_{T, P}}{\theta_{T_{o}, P_{o}}}
$$

where $\theta$ is the dial reading and $T_{o}$ and $P_{o}$ are the relative temperature and pressure, respectively. Combining with Arrhenius Relation, the RDR model is rewritten as:

$$
R D R(T, P, \gamma)=C_{1}(P, \gamma) \exp \left(\left(C_{2}(P, \gamma)\right) / T\right\rfloor
$$

where $C_{1}$ and $C_{2}$ are model coefficients that are dependent on the pressure and shear rate.

Using the RDR approach, an accurate HTHP rheological model was developed at eight specific shear rates under HTHP conditions $\left(50-240^{\circ} \mathrm{F}, 0-8000 \mathrm{psig}\right)$ for an ester-based drilling fluid. Similarly, Demirdal [14] applied the RDR approach to establish an accurate HTHP rheological model for ester-based mud under HTHP conditions $\left(40-280^{\circ} \mathrm{F}\right.$, 500-12000psig). As for the RDR modeling strategy, it is not necessary to use a rheological model as the base equation and, thus, the assumption of base rheological model can be avoided.

Previous RDR modeling studies focused mainly on ester- and synthetic-based drilling fluids and, consequently, the implication of RDR approach on the model applicability and the predictive accuracy in non-aqueous drilling fluids, is increasingly coming to light. In contrast, the RDR approach involved in HTHP rheological modeling for water-based drilling fluid has not been reported so far. Herein, the RDR approach was utilized to reveal its applicability of developing a highly accurate HTHP rheological equation in a weighted sulphonated water-based drilling fluid. This work on the RDR application in the water-based drilling fluid is complementary with respect to non-aqueous drilling fluids, which can not only determine a relation that relates RDRs with temperature, pressure, and shear rate, but also verify the validity of RDR approach as a novel HTHP rheological modeling tool for particular drilling fluids.

\section{METHODOLOGY}

\subsection{Experimental Materials}

The sulphonated water-based drilling fluid, as a common kind of HTHP drilling fluid, was typically employed in the oil and gas industry. Such fluid has a high density of $1.5 \mathrm{~g} / \mathrm{ml}$, and consists of at least 7 functional additives such as hydrophilic solid phase, $\mathrm{pH}$ control agent, loss control agent, viscosifier, inhibitor, antioxidant, and weighting material was employed here, as shown in Table 1 . The drilling fluid was heat-aged at $180^{\circ} \mathrm{C}$ for 16 hours before testing.

Table 1. Components of the sulphonated water-based fluid referred here.

\begin{tabular}{|c|c|c|}
\hline Additives & Components & Content (wt.\%) \\
\hline-- & Water & Clay \\
\hline-- & Sodium hydroxide & $4 \sim 5$ \\
\hline pH-adjusting agent & PAMS copolymer & 0.5 \\
\hline viscosifier & Amphoteric sulfonated polymer \\
\hline viscosifier & Sulfonated phenolic resin \\
\hline Filtrate loss reducer & Lignosulfonate & 0.5 \\
\hline Filtrate loss reducer & Barite \\
\hline Weighting material & 4 \\
\hline
\end{tabular}

\subsection{Examination on Rheological Data}

Pressures applied in the tests were $0.1,15,25,55,85$, and $100 \mathrm{MPa}$. Temperatures used during the tests were 30,60 , 90,120 , and $150^{\circ} \mathrm{C}$. At each combination of pressure and temperature, the rheological data(dial readings) of the weighted sulphonated drilling fluid were measured at six specific shear rates 600, 300, 200, 100, 6, and 3rpm, using a Fann IX77 HTHP Rheometer.

To establish an accurate equation, both statistics and detailed sample selection are essential. In principle, the more the testing data, the more accurate the final formula. Although the statistic data seem to be not enough, the RDR 
approach was previously demonstrated to be accurate in defining a HTHP rheological model even with limited parameters $[13,14]$.

\subsection{Modeling Procedure}

The RDR approach was employed to determine a single relation that relates dial readings with shear rate, temperature, and pressure. The standard procedure of RDR modeling contains relative dial reading, Arrhenius relation, polynomial of pressure coefficients, and polynomial of shear rate coefficients, as shown in Fig. (1). To determine such an equation, non-linear and linear regression analysis has been used by a commercial software STATISTIC. Note that, on account of the requirements of accuracy, different expressions like logarithmic, exponential, and polynomial model can be obtained upon fitting.

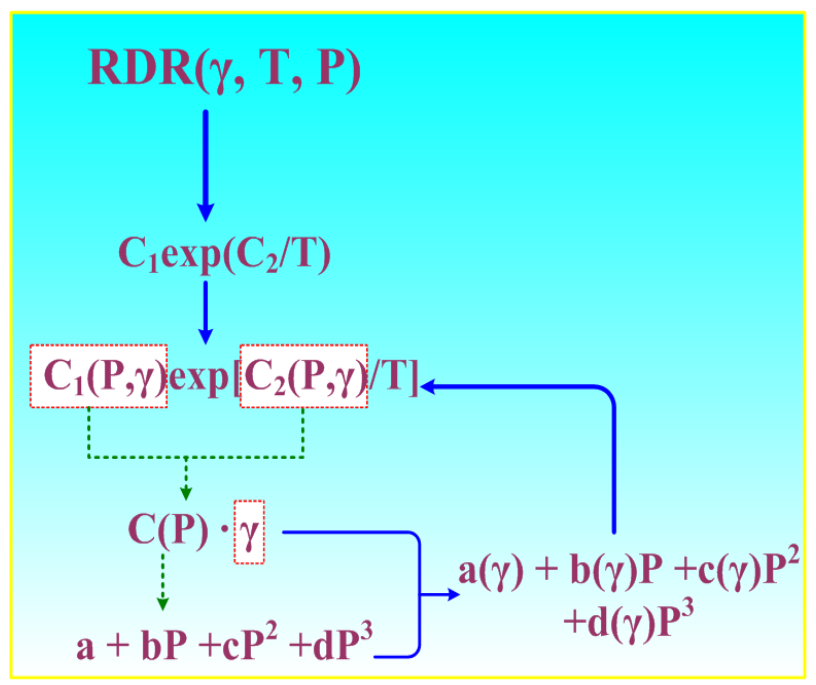

Fig. (1). HTHP rheological Modeling procedure of the RDR approach.

\section{RESULTS AND DISCUSSION}

\subsection{Relative Procedure of Dial Readings}

To reduce the effect of $\mathrm{T}$ and $\mathrm{P}$, a relative treatment was performed on dial reading data. Herein, RDR is defined as the ratio of the dial reading determined at any temperature and pressure to the dial reading determined at $0.1 \mathrm{MPa}$ and $30^{\circ} \mathrm{C}$, shown as follows:

$$
R D R=\tau / \tau_{0.1 M P a, 30^{\circ} \mathrm{C}}
$$

Table 2 collects the dial readings and RDRs under the considered conditions. As might be anticipated, the waterbased fluid is rarely pressure-dependent and highly temperature-dependent. Meanwhile, pressure effects on rheology can slightly rise at high temperature and low shear rates.

Table 2. Dial readings measured under the considered HPHT conditions ${ }^{\text {a, }}$.

\begin{tabular}{|c|c|c|c|c|c|c|c|}
\hline $\mathbf{P}$ & $\mathbf{T}$ & \multicolumn{6}{|c|}{ Shear Rates (rpm) } \\
\cline { 3 - 8 }$(\mathbf{M P a})$ & $\left({ }^{\circ} \mathbf{C}\right)$ & $\mathbf{6 0 0}$ & $\mathbf{3 0 0}$ & $\mathbf{2 0 0}$ & $\mathbf{1 0 0}$ & $\mathbf{6}$ & $\mathbf{3}$ \\
\hline 100 & 60 & $116(0.5577)$ & $87(0.5241)$ & $77(0.5238)$ & $66(0.5410)$ & $53(0.5761)$ & $53(0.6023)$ \\
\hline 100 & 90 & $68(0.3269)$ & $47(0.2831)$ & $39(0.2653)$ & $32(0.2623)$ & $25(0.2717)$ & $24(0.2727)$ \\
\hline 100 & 120 & $49(0.2356)$ & $31(0.1867)$ & $25(0.1701)$ & $18(0.1475)$ & $13(0.1413)$ & $13(0.1477)$ \\
\hline 100 & 150 & $38(0.1827)$ & $21(0.1265)$ & $16(0.1088)$ & $11(0.0902)$ & $7(0.0761)$ & $7(0.0795)$ \\
\hline 85 & 60 & $117(0.5625)$ & $88(0.5301)$ & $78(0.5306)$ & $67(0.5492)$ & $54(0.5870)$ & $53(0.6023)$ \\
\hline 85 & 90 & $68(0.3269)$ & $47(0.2831)$ & $39(0.2653)$ & $32(0.2623)$ & $25(0.2717)$ & $24(0.2727)$ \\
\hline 85 & 120 & $49(0.2356)$ & $31(0.1867)$ & $25(0.1701)$ & $18(0.1475)$ & $13(0.1413)$ & $13(0.1477)$ \\
\hline 85 & 150 & $38(0.1827)$ & $21(0.1265)$ & $16(0.1088)$ & $11(0.0902)$ & $8(0.0870)$ & $7(0.0795)$ \\
\hline
\end{tabular}


(Table $\square$ ) contd.....

\begin{tabular}{|c|c|c|c|c|c|c|c|}
\hline \multirow{2}{*}{$\begin{array}{c}\mathbf{P} \\
(\mathbf{M P a})\end{array}$} & \multirow{2}{*}{$\begin{array}{c}\mathbf{T} \\
\left({ }^{\circ} \mathbf{C}\right)\end{array}$} & \multicolumn{6}{|c|}{ Shear Rates (rpm) } \\
\hline & & 600 & 300 & 200 & 100 & 6 & 3 \\
\hline 55 & 60 & $117(0.5625)$ & $87(0.5241)$ & $77(0.5238)$ & $66(0.5410)$ & $53(0.5761)$ & $52(0.5909)$ \\
\hline 55 & 90 & $68(0.3269)$ & $47(0.2831)$ & $40(0.2721)$ & $33(0.2705)$ & $25(0.2717)$ & $24(0.2727)$ \\
\hline 55 & 120 & $49(0.2356)$ & $31(0.1867)$ & $26(0.1769)$ & $19(0.1557)$ & $14(0.1522)$ & $13(0.1477)$ \\
\hline 55 & 150 & $38(0.1827)$ & $22(0.1325)$ & $17(0.1156)$ & $12(0.0984)$ & $8(0.0870)$ & $7(0.0795)$ \\
\hline 25 & 60 & $117(0.5625)$ & $87(0.5241)$ & $77(0.5238)$ & $66(0.5410)$ & $53(0.5761)$ & $52(0.5909)$ \\
\hline 25 & 90 & $68(0.3269)$ & $47(0.2831)$ & $40(0.2721)$ & $33(0.2705)$ & $25(0.2717)$ & $24(0.2727)$ \\
\hline 25 & 120 & $49(0.2356)$ & $31(0.1867)$ & $26(0.1769)$ & $19(0.1557)$ & $14(0.1522)$ & $13(0.1477)$ \\
\hline 25 & 150 & $38(0.1827)$ & $22(0.1325)$ & $17(0.1156)$ & $12(0.0984)$ & $7(0.0761)$ & $7(0.0795)$ \\
\hline 15 & 60 & $115(0.5529)$ & $86(0.5181)$ & $76(0.5170)$ & $65(0.5328)$ & $53(0.5761)$ & $52(0.5909)$ \\
\hline 15 & 90 & $68(0.3269)$ & $47(0.2831)$ & $40(0.2721)$ & $33(0.2705)$ & $25(0.2717)$ & $24(0.2727)$ \\
\hline 15 & 120 & $49(0.2356)$ & $31(0.1867)$ & $25(0.1701)$ & $18(0.1475)$ & $14(0.1522)$ & $13(0.1477)$ \\
\hline 15 & 150 & $38(0.1827)$ & $22(0.1325)$ & $17(0.1156)$ & $12(0.0984)$ & $8(0.0870)$ & $8(0.0909)$ \\
\hline 0.1 & 30 & $208(1)$ & $166(1)$ & $147(1)$ & $122(1)$ & $92(1)$ & $88(1)$ \\
\hline
\end{tabular}

${ }^{a} T$ and $P$ separately correspond to $30^{\circ} \mathrm{C}$ and $0.1 \mathrm{MPa}$.

${ }^{\mathrm{b}}$ Data in parenthesis correspond to RDRs.

\subsection{Arrhenius Relation Between RDRs and Temperature}

Arrhenius relation is considered to be appropriate to relate RDR to temperature. $R D R(T)$ in terms of the investigated parameters can be shown as follows:

$$
R D R(T)=C_{1} \exp C_{2}(T) / T
$$

wherein $C_{I}$ and $C_{2}$ are coefficients of Arrhenius relation.

Table 3 presents the coefficient analysis of RDR vs. $T$ for Arrhenius relation. It is clear that all of the correlations $\left(R^{2}\right)$ are larger than 0.98 , and the average value is $0.9886 \pm 0.0035$, suggesting a high predictive accuracy for Arrhenius relation.

Table 3. Coefficient analysis of $R D R$ vs. $T$ for Arrhenius relation.

\begin{tabular}{|c|c|c|c|c|}
\hline$P(\mathrm{MPa})$ & $\gamma(\mathrm{rpm})$ & $C_{1}$ & $C_{2}$ & $R^{2}$ \\
\hline \multirow[t]{6}{*}{100} & 3 & 0.0375 & 167.2237 & 0.9857 \\
\hline & 6 & 0.0376 & 164.3890 & 0.9807 \\
\hline & 100 & 0.0423 & 153.5008 & 0.9861 \\
\hline & 200 & 0.0528 & 138.1225 & 0.9900 \\
\hline & 300 & 0.0643 & 126.4127 & 0.9892 \\
\hline & 600 & 0.0976 & 104.9750 & 0.9953 \\
\hline \multirow[t]{6}{*}{85} & 3 & 0.0375 & 167.2237 & 0.9857 \\
\hline & 6 & 0.0385 & 164.0179 & 0.9865 \\
\hline & 100 & 0.0416 & 155.4050 & 0.9873 \\
\hline & 200 & 0.0521 & 139.7473 & 0.9909 \\
\hline & 300 & 0.0635 & 127.8266 & 0.9901 \\
\hline & 600 & 0.0967 & 106.0262 & 0.9958 \\
\hline \multirow[t]{6}{*}{55} & 3 & 0.0383 & 164.7892 & 0.9842 \\
\hline & 6 & 0.0413 & 158.7604 & 0.9857 \\
\hline & 100 & 0.0469 & 147.3398 & 0.9859 \\
\hline & 200 & 0.0572 & 133.3671 & 0.9897 \\
\hline & 300 & 0.0658 & 124.9897 & 0.9917 \\
\hline & 600 & 0.0967 & 106.0262 & 0.9958 \\
\hline \multirow[t]{6}{*}{25} & 3 & 0.0383 & 164.7892 & 0.9842 \\
\hline & 6 & 0.0395 & 161.4868 & 0.9808 \\
\hline & 100 & 0.0469 & 147.3398 & 0.9859 \\
\hline & 200 & 0.0572 & 133.3671 & 0.9897 \\
\hline & 300 & 0.0658 & 124.9897 & 0.9917 \\
\hline & 600 & 0.0967 & 106.0262 & 0.9958 \\
\hline
\end{tabular}


(Table $\square$ ) contd.....

\begin{tabular}{|c|c|c|c|c|}
\hline$P(\mathbf{M P a})$ & $\gamma($ rpm $)$ & $C_{I}$ & $C_{2}$ & $R^{2}$ \\
\hline \multirow[t]{6}{*}{15} & 3 & 0.0401 & 161.9553 & 0.9885 \\
\hline & 6 & 0.0413 & 158.7615 & 0.9857 \\
\hline & 100 & 0.0461 & 147.5490 & 0.9837 \\
\hline & 200 & 0.0565 & 133.3969 & 0.9889 \\
\hline & 300 & 0.0667 & 123.5629 & 0.9908 \\
\hline & 600 & 0.985 & 103.9176 & 0.9947 \\
\hline
\end{tabular}

\subsection{Modeling of Coefficient $C_{1}$ and $C_{2}$ vs. $P$}

Once the relation between $R D R$ and $T$ was determined, the next step was to determine whether coefficients of the model $\left(C_{1}\right.$ and $\left.C_{2}\right)$ are dependent on $P$ and $\gamma$. Table 4 shows that both $C_{1}$ and $C_{2}$ are functions of $P$ and $\gamma$. If these coefficients are defined as the functions of $P$ and $\gamma, R D R(T, P, \gamma)$ can be written as (5).

In order to get a general expression, the regression analysis was carried out to determine the effect of $P$ and $\gamma$ on these constants $\left(C_{1}\right.$ and $\left.C_{2}\right)$. Herein, the effect of $P$ on $C_{1}$ and $C_{2}$ under constant $\gamma$ was analysed. Third degree polynomial defines the relation of $C_{1}$ and $C_{2} v s . P$ as:

$$
\begin{aligned}
& C_{1}(P)=a_{1}+b_{1} P+c_{1} P^{2}+d_{1} P^{3} \\
& C_{2}(P)=a_{2}+b_{2} P+c_{2} P^{2}+d_{2} P^{3}
\end{aligned}
$$

wherein $a, b, c$, and $d$ are the model coefficients.

\begin{tabular}{|c|c|c|c|c|c|c|}
\hline \multirow{2}{*}{ Coefficients } & \multicolumn{6}{|c|}{$C_{I}(P)$} \\
\hline & 3 & 6 & 100 & 200 & 300 & 600 \\
\hline$a_{1}$ & 0.73 & 0.72 & 0.31 & 0.20 & 0.13 & 0.18 \\
\hline$b_{1}$ & $-4.0 \times 10^{-4}$ & $-4.0 \times 10^{-4}$ & $-5.0 \times 10^{-5}$ & $-8.6 \mathrm{E}-6$ & $4.0 \times 10^{-5}$ & $2.0 \times 10^{-5}$ \\
\hline$c_{1}$ & $-6.5 \times 10^{-8}$ & $-6.4 \times 10^{-8}$ & $-1.2 \times 10^{-8}$ & $-2.9 \times 10^{-9}$ & $6.0 \times 10^{-9}$ & $3.9 \times 10^{-10}$ \\
\hline$d l$ & $-3.2 \times 10^{-12}$ & $-3.2 \times 10^{-12}$ & $-7.8 \times 10^{-13}$ & $-1.5 \times 10^{-13}$ & $2.8 \times 10^{-13}$ & $-1.5 \times 10^{-14}$ \\
\hline \multirow[t]{2}{*}{$R^{2}$} & 0.998 & 0.997 & 0.999 & 0.995 & 0.997 & 0.998 \\
\hline & \multicolumn{6}{|c|}{$C_{2}(P)$} \\
\hline$a_{2}$ & -251.34 & 41.66 & 11.75 & 50.18 & 75.78 & 74.46 \\
\hline$b_{2}$ & -0.17 & 0.04 & $-1.5 \times 10^{-2}$ & $-2.7 \times 10^{-3}$ & 0.01 & $5.5 \times 10^{-3}$ \\
\hline$c_{2}$ & $-3.0 \times 10^{-5}$ & $4.1 \times 10^{-6}$ & $-2.0 \times 10^{-6}$ & $-9.7 \times 10^{-8}$ & $2.1 \times 10^{-6}$ & $9.8 \times 10^{-7}$ \\
\hline$d_{2}$ & $-1.5 \times 10^{-9}$ & $3.0 \times 10^{-11}$ & $-9.9 \times 10^{-11}$ & $-7.0 \times 10^{-12}$ & $9.7 \times 10^{-11}$ & $3.7 \times 10^{-11}$ \\
\hline$\overline{R^{2}}$ & 0.996 & 0.995 & 0.995 & 0.996 & 0.994 & 0.996 \\
\hline
\end{tabular}

Table 4. Coefficient analysis of $C_{1}$ and $C_{2} v s . P$ for third degree polynomial relation.

It can be readily found that coefficients of multiple determination $\left(R^{2}\right)$ for $C_{1}(P)$ and $C_{2}(P)$ are all close to 1.0 , which reveals significant correlation between the actual and predictive values. Apparently, the proposed third degree polynomial is quite suitable to fit $C(P)$. Next, $C_{I}(P)$ and $C_{2}(P)$ were further used to establish $C_{l}(P, \gamma)$ and $C_{2}(P, \gamma)$.

\subsection{Relation Betwen Coefficients of $C_{1}(P)$ and $C_{2}(P)$ and $\gamma$}

In order to determine $C_{l}(P, \gamma)$ and $C_{2}(P, \gamma)$, the effect of $\gamma$ on coefficients $(a, b, c$, and $d)$ shown in $C(P)$ should be determined. Non-linear regression analysis was carried out using either one-parameter or two-parameter curves. The relations between the coefficients of $C_{1}(P)$ and $C_{2}(P)$ and $\gamma$ are presented in Table 5.

Table 5. Relations between coefficients of $C(P)$ and $\gamma$.

\begin{tabular}{|c|c|c|}
\hline $\boldsymbol{C}(\boldsymbol{P})$ & Equations of Coefficients of $\boldsymbol{C}(\boldsymbol{P}) \boldsymbol{v s} \cdot \boldsymbol{\gamma}$ & $\boldsymbol{R}^{2}$ \\
\hline$C_{1}$ & $a=(1.32073+0.02104 \gamma)^{1 / 1.06571}$ & 0.998 \\
\hline & $b=-0.00065+0.00012 \ln (\gamma+5.37232)$ & 0.986 \\
\hline & $c=\left(-1.1431 \times 10^{-7}\right)-2.1299 \times 10^{-8}(\gamma+6.18217)$ & 0.992 \\
\hline & $d=\left(-6.3934 \times 10^{-12}\right)-2.1299 \times 10^{-8}(\gamma+11.02309)$ & 0.996 \\
\hline$C_{2}$ & $a=6.80525+7.38181 \ln (\gamma-3)$ & 0.983 \\
\hline
\end{tabular}


(Table $\square$ ) contd......

\begin{tabular}{|c|c|c|c|}
\hline Table $\square$ contd..... & Equations of Coefficients of $\boldsymbol{C}(\boldsymbol{P})$ vs. $\boldsymbol{\gamma}$ & $\boldsymbol{R}^{\mathbf{2}}$ \\
\hline $\boldsymbol{C}(\boldsymbol{P})$ & $b=0.0133+0.004351 \ln (\gamma-3)$ & 0.894 \\
\hline & $c=\left(-2.3171 \times 10^{-6}\right)+7.6818 \times 10^{-7} \ln (\gamma-3)$ & 0.942 \\
\hline & $d=\left(-1.5396 \times 10^{-10}\right)+3.7431 \times 10^{-11} \ln (\gamma-3)$ & 0.978 \\
\hline
\end{tabular}

Listed in Table 5 are the relation that well relate coefficient of $\mathrm{C}(\mathrm{p})$ to shear rate. For all relation, $R^{2}$ values are almost equal to 0.9 , and average value is as high as $0.971 \pm 0.027$, suggesting a good relation between the predictive and actual values. Substituting relations listed in Table 5 to $C_{l}(P)$ and $C_{2}(P)$, the coefficients $C$ can be shown as a function of $P$ and $\gamma$ as follows:

$$
\begin{aligned}
C_{1}(P, \gamma)= & (1.32073+0.02104 \gamma)^{\frac{1}{1.06571}}+[-0.00065+0.00012 \ln (\gamma \\
& +5.37232)] P+\left[1.1431 \times 10^{-7}+2.1299 \times 10^{-8}(\gamma+6.18217)\right] P^{2} \\
& -\left[6.3934 \times 10^{-12}+2.1299 \times 10^{-8}(\gamma+11.02309)\right] P^{3} \\
C_{2}(P, \gamma)= & 6.80525+7.3818 \ln (\gamma-3)-[0.0133+0.00435 \ln (\gamma-3)] P \\
& +\left[-2.3171 \times 10^{-6}+7.6818 \times 10^{-7} \ln (\gamma-3)\right] P^{2}+[1.5396 \\
& \left.\times 10^{-10}-3.7431 \times 10^{-11} \ln (\gamma-3)\right] P^{3}
\end{aligned}
$$

These two Eq.11 and Eq.12 achieved above can be further substituted into $R D R(T)$. As a consequence, a function relating RDR to $T, P$, and $\gamma$ can be determined by using one equation (see Eq. 7).

\subsection{Analysis on Deviation of RDR Rheolical Model}

Analysis on the predictive deviation of HTHP rheological model developed here was further carried out to evaluate its applicability. Table $\mathbf{6}$ lists main results, including the tested and calculated dial readings, residual, and absolute error.

\begin{tabular}{|c|c|c|c|c|c|c|c|}
\hline \multirow{2}{*}{ No } & \multicolumn{3}{|c|}{ Independent Variables } & \multicolumn{2}{|c|}{ Dial Readings } & \multirow{2}{*}{ Residual } & \multirow{2}{*}{$\begin{array}{c}\text { Absolute Error } \\
(\%)\end{array}$} \\
\hline & $P(\mathrm{MPa})$ & $T\left({ }^{\circ} \mathrm{C}\right)$ & $\gamma($ rpm $)$ & Factual & Calculated & & \\
\hline 1 & 100 & 60 & 3 & 0.6023 & 0.6037 & $-1.39 \times 10^{-3}$ & 0.23 \\
\hline 2 & 100 & 60 & 6 & 0.5761 & 0.5676 & $8.50 \times 10^{-3}$ & 1.48 \\
\hline 3 & 100 & 60 & 100 & 0.5410 & 0.5279 & $1.31 \times 10^{-2}$ & 2.42 \\
\hline 4 & 100 & 60 & 200 & 0.5238 & 0.5223 & $1.55 \times 10^{-3}$ & 0.30 \\
\hline 5 & 100 & 60 & 300 & 0.5241 & 0.5281 & $-4.04 \times 10^{-3}$ & 0.77 \\
\hline 6 & 100 & 60 & 600 & 0.5577 & 0.5581 & $-3.83 \times 10^{-4}$ & 0.07 \\
\hline 7 & 100 & 90 & 3 & 0.2727 & 0.2734 & $-6.30 \times 10^{-4}$ & 0.23 \\
\hline 8 & 100 & 90 & 6 & 0.2717 & 0.2583 & $1.34 \times 10^{-2}$ & 4.93 \\
\hline 9 & 100 & 90 & 100 & 0.2623 & 0.2470 & $1.53 \times 10^{-2}$ & 5.82 \\
\hline 10 & 100 & 90 & 200 & 0.2653 & 0.2647 & $5.90 \times 10^{-4}$ & 0.22 \\
\hline 11 & 100 & 90 & 300 & 0.2831 & 0.2878 & $-4.69 \times 10^{-3}$ & 1.65 \\
\hline 12 & 100 & 90 & 600 & 0.3269 & 0.3267 & $1.88 \times 10^{-4}$ & 0.06 \\
\hline 13 & 100 & 120 & 3 & 0.1477 & 0.1469 & $8.73 \times 10^{-4}$ & 0.59 \\
\hline 14 & 100 & 120 & 6 & 0.1413 & 0.1367 & $4.64 \times 10^{-3}$ & 3.29 \\
\hline 15 & 100 & 120 & 100 & 0.1475 & 0.1372 & $1.03 \times 10^{-2}$ & 6.98 \\
\hline 16 & 100 & 120 & 200 & 0.1701 & 0.1613 & $8.73 \times 10^{-3}$ & 5.14 \\
\hline 17 & 100 & 120 & 300 & 0.1867 & 0.1881 & $-1.39 \times 10^{-3}$ & 0.75 \\
\hline 18 & 100 & 120 & 600 & 0.2356 & 0.2336 & $1.94 \times 10^{-3}$ & 0.82 \\
\hline
\end{tabular}
The absolute error percentages vary in the range of $0 \sim 12 \%$, and the average error percentage is calculated as 3.3397 \pm 2.2006 , which reflects high predictive accuracy for the developed HTHP rheological equation within certain temperature and pressure range.

Table 6. Analysis on deviation of the HTHP rheological model developed for the weighted sulphonated water-based drilling fluid. 
(Table $\square$ ) contd....

\begin{tabular}{|c|c|c|c|c|c|c|c|}
\hline \multirow{2}{*}{ No } & \multicolumn{3}{|c|}{ Independent Variables } & \multicolumn{2}{|c|}{ Dial Readings } & \multirow{2}{*}{ Residual } & \multirow{2}{*}{$\begin{array}{c}\text { Absolute Error } \\
(\%)\end{array}$} \\
\hline & $P(\mathbf{M P a})$ & $T\left({ }^{\circ} \mathrm{C}\right)$ & $\gamma(\mathbf{r p m})$ & Factual & Calculated & & \\
\hline 19 & 100 & 150 & 3 & 0.0795 & 0.0823 & $-2.74 \times 10^{-3}$ & 3.44 \\
\hline 20 & 100 & 150 & 6 & 0.0761 & 0.0729 & $3.17 \times 10^{-3}$ & 4.17 \\
\hline 21 & 100 & 150 & 100 & 0.0902 & 0.0801 & $1.01 \times 10^{-2}$ & 11.20 \\
\hline 22 & 100 & 150 & 200 & 0.1088 & 0.1062 & $2.67 \times 10^{-3}$ & 2.46 \\
\hline 23 & 100 & 150 & 300 & 0.1265 & 0.1334 & $-6.90 \times 10^{-3}$ & 5.45 \\
\hline 24 & 100 & 150 & 600 & 0.1827 & 0.1839 & $-1.16 \times 10^{-3}$ & 0.64 \\
\hline 25 & 85 & 60 & 3 & 0.6023 & 0.6014 & $8.35 \times 10^{-4}$ & 0.14 \\
\hline 26 & 85 & 60 & 6 & 0.5870 & 0.5795 & $7.41 \times 10^{-3}$ & 1.26 \\
\hline 27 & 85 & 60 & 100 & 0.5492 & 0.5367 & $1.25 \times 10^{-2}$ & 2.27 \\
\hline 28 & 85 & 60 & 200 & 0.5306 & 0.5264 & $4.22 \times 10^{-3}$ & 0.79 \\
\hline 29 & 85 & 60 & 300 & 0.5301 & 0.5305 & $-4.15 \times 10^{-4}$ & 0.08 \\
\hline 30 & 85 & 60 & 600 & 0.5625 & 0.5604 & $2.07 \times 10^{-3}$ & 0.37 \\
\hline 31 & 85 & 90 & 3 & 0.2727 & 0.2733 & $-5.90 \times 10^{-4}$ & 0.22 \\
\hline 32 & 85 & 90 & 6 & 0.2717 & 0.2650 & $6.76 \times 10^{-3}$ & 2.49 \\
\hline 33 & 85 & 90 & 100 & 0.2623 & 0.2527 & $9.57 \times 10^{-3}$ & 3.65 \\
\hline 34 & 85 & 90 & 200 & 0.2653 & 0.2661 & $-7.54 \times 10^{-4}$ & 0.28 \\
\hline 35 & 85 & 90 & 300 & 0.2831 & 0.2875 & $-4.35 \times 10^{-3}$ & 1.54 \\
\hline 36 & 85 & 90 & 600 & 0.3269 & 0.3271 & $-2.25 \times 10^{-4}$ & 0.07 \\
\hline 37 & 85 & 120 & 3 & 0.1477 & 0.1475 & $2.69 \times 10^{-4}$ & 0.18 \\
\hline 38 & 85 & 120 & 6 & 0.1413 & 0.1434 & $-2.06 \times 10^{-3}$ & 1.46 \\
\hline 39 & 85 & 120 & 100 & 0.1475 & 0.1416 & $5.89 \times 10^{-3}$ & 3.99 \\
\hline 40 & 85 & 120 & 200 & 0.1701 & 0.1624 & $7.71 \times 10^{-3}$ & 4.53 \\
\hline 41 & 85 & 120 & 300 & 0.1867 & 0.1879 & $-1.13 \times 10^{-3}$ & 0.60 \\
\hline 42 & 85 & 120 & 600 & 0.2356 & 0.2337 & $1.84 \times 10^{-3}$ & 0.78 \\
\hline 43 & 85 & 150 & 3 & 0.0795 & 0.0831 & $-3.57 \times 10^{-3}$ & 4.49 \\
\hline 44 & 85 & 150 & 6 & 0.0870 & 0.0807 & $6.25 \times 10^{-3}$ & 7.18 \\
\hline 45 & 85 & 150 & 100 & 0.0902 & 0.0838 & $6.38 \times 10^{-3}$ & 7.08 \\
\hline 46 & 85 & 150 & 200 & 0.1088 & 0.1074 & $1.44 \times 10^{-3}$ & 1.32 \\
\hline 47 & 85 & 150 & 300 & 0.1265 & 0.1337 & $-7.23 \times 10^{-3}$ & 5.72 \\
\hline 48 & 85 & 150 & 600 & 0.1827 & 0.1840 & $-1.30 \times 10^{-3}$ & 0.71 \\
\hline 49 & 55 & 60 & 3 & 0.5909 & 0.5924 & $-1.51 \times 10^{-3}$ & 0.26 \\
\hline 50 & 55 & 60 & 6 & 0.5761 & 0.5785 & $-2.41 \times 10^{-3}$ & 0.42 \\
\hline 51 & 55 & 60 & 100 & 0.5410 & 0.5409 & $4.56 \times 10^{-5}$ & 0.01 \\
\hline 52 & 55 & 60 & 200 & 0.5238 & 0.5252 & $-1.42 \times 10^{-3}$ & 0.27 \\
\hline 53 & 55 & 60 & 300 & 0.5241 & 0.5282 & $-4.13 \times 10^{-3}$ & 0.79 \\
\hline 54 & 55 & 60 & 600 & 0.5625 & 0.5647 & $-2.20 \times 10^{-3}$ & 0.39 \\
\hline 55 & 55 & 90 & 3 & 0.2727 & 0.2721 & $6.68 \times 10^{-4}$ & 0.24 \\
\hline 56 & 55 & 90 & 6 & 0.2717 & 0.2721 & $-3.40 \times 10^{-4}$ & 0.12 \\
\hline 57 & 55 & 90 & 100 & 0.2705 & 0.2670 & $3.48 \times 10^{-3}$ & 1.29 \\
\hline 58 & 55 & 90 & 200 & 0.2721 & 0.2706 & $1.50 \times 10^{-3}$ & 0.55 \\
\hline 59 & 55 & 90 & 300 & 0.2831 & 0.2875 & $-4.41 \times 10^{-3}$ & 1.56 \\
\hline 60 & 55 & 90 & 600 & 0.3269 & 0.3274 & $-4.77 \times 10^{-4}$ & 0.15 \\
\hline 61 & 55 & 120 & 3 & 0.1477 & 0.1470 & $6.84 \times 10^{-4}$ & 0.46 \\
\hline 62 & 55 & 120 & 6 & 0.1522 & 0.1499 & $2.31 \times 10^{-3}$ & 1.52 \\
\hline 63 & 55 & 120 & 100 & 0.1557 & 0.1553 & $4.66 \times 10^{-4}$ & 0.30 \\
\hline 64 & 55 & 120 & 200 & 0.1769 & 0.1682 & $8.65 \times 10^{-3}$ & 4.89 \\
\hline 65 & 55 & 120 & 300 & 0.1867 & 0.1897 & $-2.92 \times 10^{-3}$ & 1.57 \\
\hline 66 & 55 & 120 & 600 & 0.2356 & 0.2335 & $2.12 \times 10^{-3}$ & 0.90 \\
\hline 67 & 55 & 150 & 3 & 0.0795 & 0.0821 & $-2.51 \times 10^{-3}$ & 3.16 \\
\hline 68 & 55 & 150 & 6 & 0.0870 & 0.0850 & $1.92 \times 10^{-3}$ & 2.21 \\
\hline
\end{tabular}


(Table $\square$ ) contd....

\begin{tabular}{|c|c|c|c|c|c|c|c|}
\hline \multirow{2}{*}{ No } & \multicolumn{3}{|c|}{ Independent Variables } & \multicolumn{2}{|c|}{ Dial Readings } & \multirow{2}{*}{ Residual } & \multirow{2}{*}{$\begin{array}{c}\text { Absolute Error } \\
(\%)\end{array}$} \\
\hline & $P($ MPa $)$ & $T\left({ }^{\circ} \mathrm{C}\right)$ & $\gamma(\mathbf{r p m})$ & Factual & Calculated & & \\
\hline 69 & 55 & 150 & 100 & 0.0984 & 0.0948 & $3.57 \times 10^{-3}$ & 3.63 \\
\hline 70 & 55 & 150 & 200 & 0.1156 & 0.1135 & $2.16 \times 10^{-3}$ & 1.86 \\
\hline 71 & 55 & 150 & 300 & 0.1325 & 0.1368 & $-4.32 \times 10^{-3}$ & 3.26 \\
\hline 72 & 55 & 150 & 600 & 0.1827 & 0.1840 & $-1.28 \times 10^{-3}$ & 0.70 \\
\hline 73 & 25 & 60 & 3 & 0.5909 & 0.5886 & $2.31 \times 10^{-3}$ & 0.39 \\
\hline 74 & 25 & 60 & 6 & 0.5761 & 0.5748 & $1.31 \times 10^{-3}$ & 0.23 \\
\hline 75 & 25 & 60 & 100 & 0.5410 & 0.5371 & $3.84 \times 10^{-3}$ & 0.71 \\
\hline 76 & 25 & 60 & 200 & 0.5238 & 0.5197 & $4.14 \times 10^{-3}$ & 0.79 \\
\hline 77 & 25 & 60 & 300 & 0.5241 & 0.5221 & $1.99 \times 10^{-3}$ & 0.38 \\
\hline 78 & 25 & 60 & 600 & 0.5625 & 0.5599 & $2.64 \times 10^{-3}$ & 0.47 \\
\hline 79 & 25 & 90 & 3 & 0.2727 & 0.2719 & $8.66 \times 10^{-4}$ & 0.32 \\
\hline 80 & 25 & 90 & 6 & 0.2717 & 0.2736 & $-1.90 \times 10^{-3}$ & 0.70 \\
\hline 81 & 25 & 90 & 100 & 0.2705 & 0.2694 & $1.06 \times 10^{-3}$ & 0.39 \\
\hline 82 & 25 & 90 & 200 & 0.2721 & 0.2706 & $1.48 \times 10^{-3}$ & 0.54 \\
\hline 83 & 25 & 90 & 300 & 0.2831 & 0.2866 & $-3.43 \times 10^{-3}$ & 1.21 \\
\hline 84 & 25 & 90 & 600 & 0.3269 & 0.3270 & $-3.82 \times 10^{-5}$ & 0.01 \\
\hline 85 & 25 & 120 & 3 & 0.1477 & 0.1479 & $-1.56 \times 10^{-4}$ & 0.11 \\
\hline 86 & 25 & 120 & 6 & 0.1522 & 0.1511 & $1.03 \times 10^{-3}$ & 0.68 \\
\hline 87 & 25 & 120 & 100 & 0.1557 & 0.1599 & $-4.12 \times 10^{-3}$ & 2.65 \\
\hline 88 & 25 & 120 & 200 & 0.1769 & 0.1698 & $7.03 \times 10^{-3}$ & 3.97 \\
\hline 89 & 25 & 120 & 300 & 0.1867 & 0.1899 & $-3.11 \times 10^{-3}$ & 1.66 \\
\hline 90 & 25 & 120 & 600 & 0.2356 & 0.2337 & $1.83 \times 10^{-3}$ & 0.78 \\
\hline 91 & 25 & 150 & 3 & 0.0795 & 0.0833 & $-3.71 \times 10^{-3}$ & 4.66 \\
\hline 92 & 25 & 150 & 6 & 0.0761 & 0.0850 & $-8.90 \times 10^{-3}$ & 11.70 \\
\hline 93 & 25 & 150 & 100 & 0.0984 & 0.1004 & $-2.01 \times 10^{-3}$ & 2.04 \\
\hline 94 & 25 & 150 & 200 & 0.1156 & 0.1157 & $-2.87 \times 10^{-5}$ & 0.02 \\
\hline 95 & 25 & 150 & 300 & 0.1325 & 0.1372 & $-4.68 \times 10^{-3}$ & 3.53 \\
\hline 96 & 25 & 150 & 600 & 0.1827 & 0.1841 & $-1.45 \times 10^{-3}$ & 0.79 \\
\hline 97 & 15 & 60 & 3 & 0.5909 & 0.5908 & $8.39 \times 10^{-5}$ & 0.01 \\
\hline 98 & 15 & 60 & 6 & 0.5761 & 0.5793 & $-3.21 \times 10^{-3}$ & 0.56 \\
\hline 99 & 15 & 60 & 100 & 0.5328 & 0.5361 & $-3.35 \times 10^{-3}$ & 0.63 \\
\hline 100 & 15 & 60 & 200 & 0.5170 & 0.5185 & $-1.54 \times 10^{-3}$ & 0.30 \\
\hline 101 & 15 & 60 & 300 & 0.5181 & 0.5204 & $-2.32 \times 10^{-3}$ & 0.45 \\
\hline 102 & 15 & 60 & 600 & 0.5529 & 0.5545 & $-1.57 \times 10^{-3}$ & 0.28 \\
\hline 103 & 15 & 90 & 3 & 0.2727 & 0.2726 & $1.51 \times 10^{-4}$ & 0.06 \\
\hline 104 & 15 & 90 & 6 & 0.2717 & 0.2735 & $-1.75 \times 10^{-3}$ & 0.64 \\
\hline 105 & 15 & 90 & 100 & 0.2705 & 0.2643 & $6.15 \times 10^{-3}$ & 2.27 \\
\hline 106 & 15 & 90 & 200 & 0.2721 & 0.2682 & $3.94 \times 10^{-3}$ & 1.45 \\
\hline 107 & 15 & 90 & 300 & 0.2831 & 0.2856 & $-2.45 \times 10^{-3}$ & 0.87 \\
\hline 108 & 15 & 90 & 600 & 0.3269 & 0.3267 & $2.47 \times 10^{-4}$ & 0.08 \\
\hline 109 & 15 & 120 & 3 & 0.1477 & 0.1492 & $-1.43 \times 10^{-3}$ & 0.96 \\
\hline 110 & 15 & 120 & 6 & 0.1522 & 0.1512 & $9.50 \times 10^{-4}$ & 0.62 \\
\hline 111 & 15 & 120 & 100 & 0.1475 & 0.1562 & $-8.63 \times 10^{-3}$ & 5.85 \\
\hline 112 & 15 & 120 & 200 & 0.1701 & 0.1675 & $2.55 \times 10^{-3}$ & 1.50 \\
\hline 113 & 15 & 120 & 300 & 0.1867 & 0.1886 & $-1.83 \times 10^{-3}$ & 0.98 \\
\hline 114 & 15 & 120 & 600 & 0.2356 & 0.2342 & $1.37 \times 10^{-3}$ & 0.58 \\
\hline 115 & 15 & 150 & 3 & 0.0909 & 0.0854 & $5.53 \times 10^{-3}$ & 6.09 \\
\hline 116 & 15 & 150 & 6 & 0.0870 & 0.0862 & $7.36 \times 10^{-4}$ & 0.85 \\
\hline 117 & 15 & 150 & 100 & 0.0984 & 0.0989 & $-5.46 \times 10^{-4}$ & 0.55 \\
\hline 118 & 15 & 150 & 200 & 0.1156 & 0.1137 & $1.92 \times 10^{-3}$ & 1.66 \\
\hline
\end{tabular}


(Table $\square$ ) contd.....

\begin{tabular}{|c|c|c|c|c|c|c|c|}
\hline \multirow{2}{*}{ No } & \multicolumn{3}{|c|}{ Independent Variables } & \multicolumn{2}{|c|}{ Dial Readings } & \multirow{2}{*}{ Residual } & \multirow{2}{*}{$\begin{array}{c}\text { Absolute Error } \\
(\%)\end{array}$} \\
\hline & $P($ MPa $)$ & $T\left({ }^{\circ} \mathrm{C}\right)$ & $\gamma(\mathrm{rpm})$ & Factual & Calculated & & \\
\hline 119 & 15 & 150 & 300 & 0.1325 & 0.1355 & $-2.96 \times 10^{-3}$ & 2.23 \\
\hline 120 & 15 & 150 & 600 & 0.1827 & 0.1844 & $-1.67 \times 10^{-3}$ & 0.92 \\
\hline
\end{tabular}

To gain a further insight into the relation between deviation and variables, deviation distributions with respect to $T$, $P$, and $\gamma$ is investigated. All of the results are presented in Fig. (2).
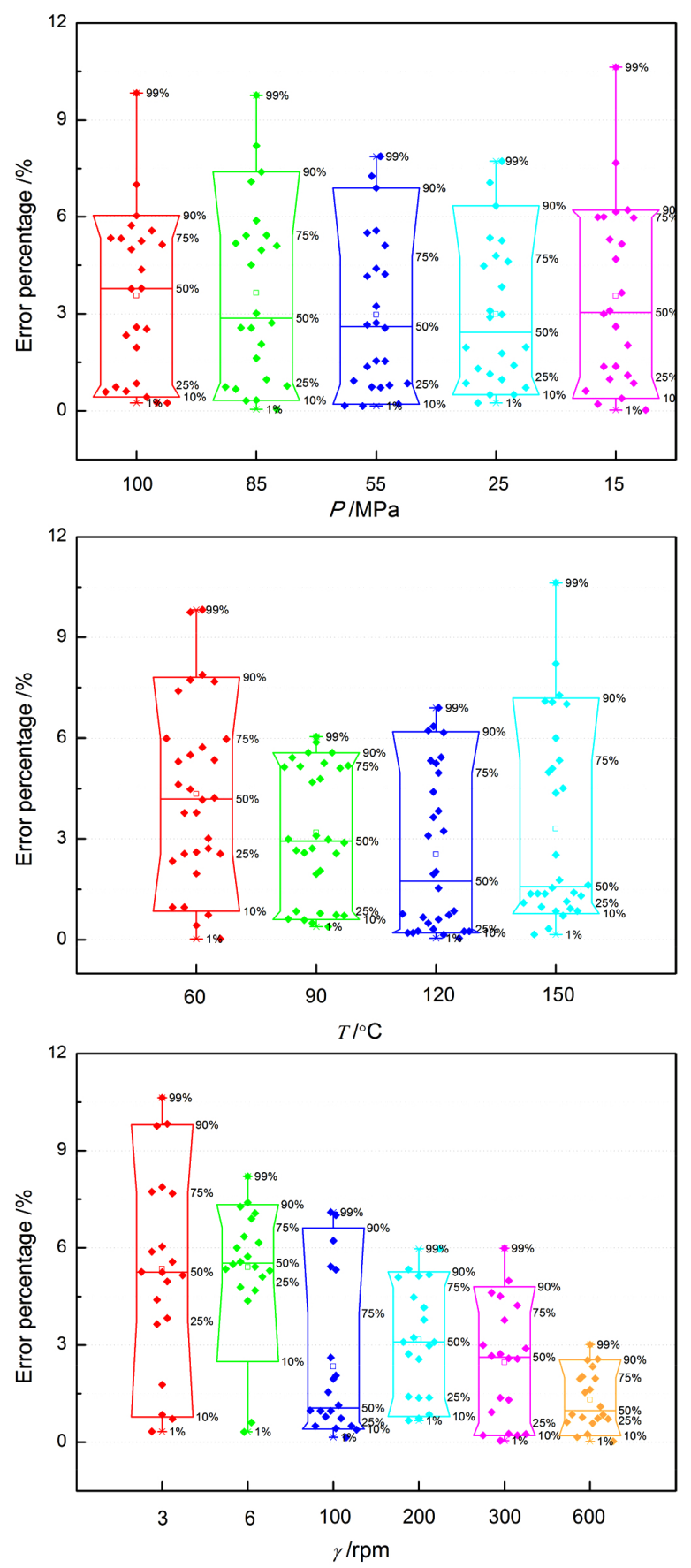

Fig. (2). Box plots of error percentage vs. $P, T$, and $\gamma$, respectively, for the RDR models, where the 10th, 25th, 50th, 75th, and 90th percentile of deviation dataset are marked. 
Fig. (2) compares distribution of deviation data related to $P$ for the RDR models. Here, the key percentiles of deviation dataset are marked in box plots. It can be readily found that the extreme deviation is about $11 \%$, and $99 \%$ deviation are less than $11 \%$, reflecting excellent prediction for the RDR model. Besides, the median line at $100 \mathrm{MPa}$ is slightly higher than others, which suggests that the predictive accuracy at $100 \mathrm{MPa}$ is lower than others. The fact that the deviation area of $85 \mathrm{MPa}$ exhibits slightly larger means a larger error distribution.

In Fig. (2), the distribution of error percentage $v s . T$ is offered. It is clear that the extreme deviation appears at $150^{\circ} \mathrm{C}$, and the highest median line at $60^{\circ} \mathrm{C}$. Meanwhile, the deviation area is longer at $60^{\circ} \mathrm{C}$ than others. These results show that the predictive accuracy is slightly lower at $60^{\circ} \mathrm{C}$ than any others.

In addition, Fig. (2) gives box plots of the distribution of error percentage $v s . \gamma$. One readily finds that the extreme deviation appears at $3 \mathrm{rpm}$, and the high median lines appear at 3 and $6 \mathrm{rpm}$. This finding reveals that the predictive accuracy should be worse at low shear rates than those at high shear rates. That is to say, the established model does not always accurately predict the measured shear stresses at low shear rates.

According to deviation analysis given above, the RDR model defined for water-based drilling fluid has a low predictive error less than $11.7 \%$. Although large errors occur at high pressure and low shear rates, they meet requirement of predictive accuracy on-site. It can be reasonably concluded that an accurate HTHP rheological model is established for the sulphonated water-based drilling fluid considered here.

\section{CONCLUSION}

Developing an accurate HTHP rheology model is complex for drilling fluids, due to the involvement of multiple variables. In the present work, the RDR experiment was conducted on the weighted sulphonated water-based drilling fluid applied in Southwest China Gas Fields, to establish an accurate HTHP rheology model at six specific shear rates $(3-600 \mathrm{rpm})$ under certain temperature $\left(30-150^{\circ} \mathrm{C}\right)$ and pressure $(0.1-150 \mathrm{MPa})$ conditions. The predictive deviation was examined to evaluate applicability of RDR approach in HTHP rheological modeling for the sulphonated waterbased drilling fluid.

The modeling procedure was discussed in detail for the purpose of validating RDR application. The determined HTHP rheological equation has a lower predictive deviation $(<11.7 \%)$, illustrating that it can relate dial readings with shear rate, temperature, and pressure very well. The larger errors readily happen at high pressure and low shear rates. This work not only develops the highly accurate HTHP rheological equation for the weighted sulphonated water-based drilling fluid, but also validates RDR application in the field of HTHP rheological modeling of drilling fluids. As an excellent modeling tool, the RDR approach can be extended to other drilling fluids, which would have large application potential in the future.

\section{CONSENT FOR PUBLICATION}

Not applicable.

\section{CONFLICT OF INTEREST}

The authors declare no conflict of interest, financial or otherwise.

\section{ACKNOWLEDGEMENTS}

The authors thank the Scientific Research Foundation of ZJOU (Project No. Q1510), Training Project Foundation of ZJOU, National Science and Technology Major Project(No. 2016ZX05060-015) and the Nature Science Foundation of China(Project No. 11472246) for financial support.

\section{REFERENCES}

[1] S.Y. Zhao, J.N. Yan, Y. Shu, and H.X. Zhang, "Rheological properties of oil-based drilling fluids at high temperature and high pressure", $J$. Cent, South Univ. Technol., vol. 15, pp. 457-461, 2008.

[http://dx.doi.org/10.1007/s11771-008-0399-7]

[2] S.Y. Zhao, J.N. Yan, Y. Shu, H.K. Li, L. Li, and T.W. Ding, "Prediction model for rheological parameters of oil-based drilling fluids at high temperature and high pressure", Acta Petrol. Sin., vol. 30, no. 4, pp. 603-606, 2009.

[3] L Wang, and Q Wu, "Study on rheological behaviors of high temperature water based drilling fluid", Drilling and production technology, vol. 32, no. 6, pp. 20-21, 2009. 
[4] H.Z. Zhao, Y.Z. Xue, G.R. Li, B.F. Liu, and H.X. Wang, "Rheological properties of high-temperature water based drilling fluids at high temperature and high pressure", Pet. Drill. Tech., vol. 37, no. 1, pp. 5-9, 2009.

[5] F.H. Wang, R.H. Wang, J.H. Liu, L. Wang, J. Li, and L.F. Che, "Rheology of high density water-based drilling fluid at high temperature and high pressure", Acta Petrol. Sin., vol. 31, no. 2, pp. 306-310, 2010.

[6] W.C. McMordie, R.B. Bennett, and R.G. Bland, "The effect of temperature and pressure on the viscosity of oil-base muds", J. Pet. Technol., pp. 884-886, 1975.

[http://dx.doi.org/10.2118/4974-PA]

[7] O.H. Houwen, and T. Geehan, "Rheology of oil-based muds", SPE Annual Technical Conference and Exhibition, New Orleans, Louisiana, 1986.

[http://dx.doi.org/10.2118/15416-MS]

[8] K.H. Hiller, "Rheological measurement on clay suspensions and drilling fluids at high temperature and pressures", J. Pet. Technol., vol. 15, no. 7 , pp. $779-788$.

[http://dx.doi.org/10.2118/489-PA]

[9] N.J. Alderman, A. Gavignet, D. Guillot, and G.C. Maitland, "High-temperature, high-pressure rheology of water-based muds", SPE Annual Technical Conference and Exhibition, 1988 Houston, Texas

[http://dx.doi.org/10.2118/18035-MS]

[10] R. Rommetveit, and K.S. Bjorkevoll, Temperature and pressure effects on drilling fluid rheology and ECD in very deep wells., SPE/IADC Middle East Drilling Technology Conference: Bahrain, 1997. [https://doi.org/10.2118/39282-MS] [http://dx.doi.org/10.2118/39282-MS]

[11] J.V. Fisk, and D.E. Jamlson, "Physical properties of drilling fluids at high temperatures and pressures", SPE Drill. Eng., vol. 341, p. 346, 1989.

[12] R.C. Minton, and P.A. Bern, Field measurement and analysis of circulating system pressure drops with low-toxicity oil-based drilling fluids., SPE/IADC Drilling Conference: Dallas, Texas, 1988. [https://doi.org/10.2118/17242-MS] [http://dx.doi.org/10.2118/17242-MS]

[13] T. Hemphill, "Prediction of rheological behavior of ester-based drilling fFluids under downhole conditions", International Petroleum Conference and Exhibition, 1996 Villahermosa, Mexico [http://dx.doi.org/10.2118/35330-MS]

[14] D. Barkim, "Study of flow of synthetic drilling fluids under elevated pressure and temperature conditions", Adv Cut Trans Stud., pp. 25-85, 2000.

(C) $2017 \mathrm{Xu}$ et al.

This is an open access article distributed under the terms of the Creative Commons Attribution 4.0 International Public License (CC-BY 4.0), a copy of which is available at: https://creativecommons.org/licenses/by/4.0/legalcode. This license permits unrestricted use, distribution, and reproduction in any medium, provided the original author and source are credited. 